\title{
Screening Phytochimique, Étude Toxicologique, Évaluation des Activités Antiplasmodiale et Antiradicalaire de la Tige Feuillée de Senna occidentalis Linn (Fabaceae)
}

\author{
Esseh K., \\ Afanyibo Y-G., \\ Laboratoire de Physiologie et Pharmacologie, Faculté des Sciences, \\ Université de Lomé, Centre de Recherche et de Formation sur les Plantes \\ Médicinales (CERFOPLAM), Université de Lomé, Togo \\ Ahama-Esseh K.Y.S., \\ Laboratoire de Physiologie et Biotechnologie Végétale, \\ Université de Lomé, Togo

$$
\begin{gathered}
\text { Idoh K., } \\
\text { Koudouvo K., } \\
\text { Agbonon A., } \\
\text { Gbeassor M., }
\end{gathered}
$$ \\ Laboratoire de Physiologie et Pharmacologie, Faculté des Sciences, \\ Université de Lomé, Centre de Recherche et de Formation sur les Plantes \\ Médicinales (CERFOPLAM), Université de Lomé, Togo
}

Doi: 10.19044/esj.2019.v15n6p411 URL:http://dx.doi.org/10.19044/esj.2019.v15n6p411

\section{Résumé}

La valorisation de la médecine traditionnelle passe par des études pharmacologiques sur les plantes médicinales. Ainsi pour lutter efficacement contre le paludisme, premier problème de santé publique au Togo, des études pharmacologiques, phytochimiques et toxicologiques ont été réalisées sur Senna occidentalis. La compilation des résultats des enquêtes ethnobotaniques effectuées au CERFOPLAM de 2010 à 2013 sur le paludisme et ses symptômes affiliés ont permis de sélectionner Senna occidentalis afin de vérifier les propriétés qui lui sont attribuées. Les différents tests ont été réalisés sur les extraits aqueux et hydro-éthanoliques 50 : 50 de la plante. L'activité antiplasmodiale in vitro a été réalisée sur une culture des parasites (Plasmodium falciparum : souche sauvage) selon le microtest optique. Le screening phytochimique a été caractérisé par la chromatographie sur couche mince suivie de tests colorimétriques. La toxicité préliminaire a été réalisée sur Artemia salina puis le potentiel antioxydant in 
vitro des extraits a été mesuré par le test de réduction du radical 2,2-diphényl1-picryl-hydrazyl et le dosage des polyphénols. Le test antiplasmodial a montré que l'extrait aqueux a une activité modérée sur le $P$. falciparum avec une $\mathrm{CI}_{50}$ de $91,08 \pm 0,61 \mu \mathrm{g} / \mathrm{mL}$ tandis que l'extrait hydro-éthanolique est très actif avec une $\mathrm{CI}_{50}$ de $1,00 \pm 0,05 \mu \mathrm{g} / \mathrm{mL}$. La drogue de référence (Quinine) présentant une $\mathrm{CI}_{50}=0,45 \pm 0,01 \mu \mathrm{g} / \mathrm{mL}$. Le screening phytochimique révèle la présence des grands groupes chimiques tels que les flavonoïdes, les tanins, les saponosides, les coumarines et les alcaloïdes qui seraient à l'origine de l'activité antiplasmodiale observée. Les tests antiradicalaires ont permis de retenir que Senna occidentalis n'est pas antioxydant et possède très peu de polyphénols. Cet aspect confirme l'état de stress que peut subir le Plasmodium falciparum vis-à-vis des différents extraits testés. Etant donné que les $\mathrm{CL}_{50}$ sont $3,31 \mathrm{mg} / \mathrm{mL}$ et $3,70 \mathrm{mg} / \mathrm{mL}$ donc très supérieures à 0,1 $\mathrm{mg} / \mathrm{mL}$, les deux extraits de plantes testés ne sont pas cytotoxiques. Tous ces résultats combinés justifient le choix des tradipraticiens dans l'utilisation de Senna occidentalis pour soigner leurs patients atteints du paludisme.

Mots-clés: Senna occidentalis, activité antiplasmodiale, activité antiradicalaire, Plasmodium falciparum 


\title{
Phytochemical Screening, Toxicological Study, Assessment of Antiplasmodial and Antiradical Activities of the Leafy Stem of Senna Occidentalis Linn (Fabaceae)
}

\author{
Esseh K., \\ Afanyibo $Y-G$., \\ Laboratoire de Physiologie et Pharmacologie, Faculté des Sciences, \\ Université de Lomé, Centre de Recherche et de Formation sur les Plantes \\ Médicinales (CERFOPLAM), Université de Lomé, Togo \\ Ahama-Esseh K.Y.S., \\ Laboratoire de Physiologie et Biotechnologie Végétale, \\ Université de Lomé, Togo
Idoh K.,
Koudouvo K.,
Agbonon A.,
Gbeassor M., \\ Laboratoire de Physiologie et Pharmacologie, Faculté des Sciences, \\ Université de Lomé, Centre de Recherche et de Formation sur les Plantes \\ Médicinales (CERFOPLAM), Université de Lomé, Togo
}

\begin{abstract}
The valorization of traditional medicine involves pharmacological researches on medicinal plants. Thus, to effectively fight malaria, the major public health problem in Togo, pharmacological, phytochemical and toxicological studies were carried out on Senna occidentalis. The compilation of the results on ethnobotanical surveys carried out at CERFOPLAM from 2010 to 2013 on malaria and its affiliated symptoms lead to selecting Senna occidentalis in order to confirm the characteristics attributed to it. Various tests were carried out on aqueous and hydroethanolic extracts 50:50 of the plant. The antiplasmodial activity in vitro was carried out on cultivated parasites (Plasmodium falciparum: wild strain) according to the optical micro-test. Phytochemical screening was characterized by thin layer chromatography followed by colorimetric tests. The preliminary toxicity was carried out on Artemia salina then the in vitro antioxidant potential of the extracts was measured by the reduction test of the 2, 2-diphenyl-1-picryl-
\end{abstract}


hydrazyl radical and the determination of the polyphenols. The antiplasmodial test showed that the aqueous extract has a moderate activity on $P$. falciparum with an $\mathrm{IC}_{50}$ of $91.08 \pm 0.61 \mu \mathrm{g} / \mathrm{mL}$ while the hydroethanolic extract is very active with an $\mathrm{IC}_{50}$ of $1,00 \pm 0.05 \mu \mathrm{g} / \mathrm{mL}$. The reference drug (Quinine) with an $\mathrm{IC}_{50}=0.45 \pm 0.01 \mu \mathrm{g} / \mathrm{mL}$. Phytochemical screening reveals the presence of large chemical groups such as flavonoids, tannins, saponosides, coumarins and alkaloids that may be responsible for the observed antiplasmodial activity. The antiradical tests have held that Senna occidentalis is not antioxidant and has got very few polyphenols. This aspect confirms the state of stress that Plasmodium falciparum can undergo against the various extracts tested. Since the $\mathrm{LC}_{50}$ 's are $3.31 \mathrm{mg} / \mathrm{mL}$ and $3.70 \mathrm{mg} /$ $\mathrm{mL}$, therefore, higher above $0.1 \mathrm{mg} / \mathrm{mL}$, the two extracts from plants which were tested are not cytotoxic. The combination of all these results justif the choice of traditional healers in using Senna occidentalis to treat their patients suffering from malaria.

Keywords: Senna occidentalis, antiplasmodial activity, antiradical activity, Plasmodium falciparum

\section{Introduction}

Le paludisme est une maladie parasitaire à transmission vectorielle qui constitue un problème majeur de santé publique dans les pays tropicaux (OMS, 2013). L'Afrique a plusieurs facteurs liés au risque de la maladie. Ces facteurs sont liés à l'existence du vecteur qui favorise la transmission du parasite et à cela il faut ajouter le climat chaud et les conditions socioéconomiques faibles qui ont un impact négatif sur le contrôle de la maladie (Cilundika et al., 2016). Au niveau mondial, le nombre de cas de paludisme est estimé à en moyenne 216 millions en 2016, contre 237 millions en 2010 et 211 millions en 2015 (OMS, 2017) ; la plupart des cas (90\%) ont été enregistrés dans la région ouest africaine, loin devant la région Asie du SudEst $(7 \%)$ et la Méditerranée orientale (2\%). Sur les 91 pays ayant rapporté des cas de paludisme indigène en 2016,15 représentent $80 \%$ du nombre de cas de paludisme dans le monde et tous, sauf l'Inde, sont en Afrique subsaharienne (OMS, 2017). Selon le même rapport de l'OMS, Plasmodium falciparum est le parasite responsable du paludisme le plus prévalent en Afrique subsaharienne ; il est en effet à l'origine de $99 \%$ des cas de paludisme estimés en 2016. En dehors de l'Afrique, $P$. vivax prédomine dans la région de l'Amérique (64\% des cas), et représente plus de $30 \%$ des cas dans la région Méditerranée orientale et plus de $40 \%$ dans la région Asie du Sud-Est de l'OMS.

Au niveau de la mortalité dans le monde, le nombre de décès dus au paludisme a été estimé à 445000 en 2016 dont la plupart sont survenus dans 
la région Afrique (91\%) de l'OMS, loin devant la région Asie du Sud-Est (6 $\%$ ). Les enfants de moins de cinq ans (un enfant en meurt toutes les 30 secondes) et les femmes enceintes représentent les couches les plus touchées par cette pandémie (OMS, 2010).

De nos jours on observe une résistance du Plasmodium lors des traitements du paludisme. Celle du Plasmodium falciparum (l'espèce la plus virulente) aux antipaludiques conventionnels est devenue la cause principale de la prévalence du paludisme (Wongsrichanalai et al., 1992a ,1992b). A cela s'ajoute la pauvreté des populations les plus touchées par la maladie (Courcoux, 2008). La résistance à l'artémisinine (antipaludéen le plus efficace actuellement) a fait son apparition à la frontière thaïlo-cambodgienne et a été confirmée par l'Organisation mondiale de la Santé (OMS, 2009).

$\mathrm{Au}$ Togo, le paludisme constitue le premier problème de santé publique, il représente en moyenne $53 \%$ des consultations externes et $50 \%$ des hospitalisations dans les formations sanitaires publiques en 2008. Le taux de mortalité hospitalière relative au paludisme est de $48 \%$ et constitue la maladie occupant le $1^{\mathrm{er}}$ rang des pathologies avec une létalité moyenne de $4 \%$. (PNLP, 2009). Cette situation perdure car 882430 cas avec 1361 décès ont été constatés en 2013 (PNLP, 2014). Pour se soigner, des patients se tournent également vers la médecine traditionnelle (OMS, 2002) dont les plantes constituent une grande richesse dans le traitement du paludisme. Au Togo en particulier, plusieurs études ont été faites dans le recensement des plantes utilisées pour soigner le paludisme et ses symptômes associés (Koudouvo, 2006 ; 2009 ; Dénou, 2011 ; Esseh, 2012 ; Agbodéka, 2017a), par contre peu d'études pharmacologiques ont été effectuées.

La recrudescence des cas de paludisme dans le monde ainsi que la résistance observée face aux antipaludiques recommandés actuellement par l'OMS, amènent les chercheurs à rechercher de nouvelles molécules antipaludiques plus efficaces sur le Plasmodium et moins toxiques à l'organisme humain. C'est dans cette optique que cette étude se veut de contribuer à la valorisation des plantes recensées au Togo contre le paludisme et ses symptômes associés en vue d'une lutte plus efficace et durable contre cette parasitose. Pour cela Senna occidentalis Linn a été sélectionnée pour des études antiplasmodiale, antioxydante, toxicologique ainsi que le screening phytochimique.

\section{Matériel et Méthodes}

\subsection{Réactifs}

Tous les solvants utilisés sont obtenus au laboratoire de Physiologie et de Pharmacologie; au Centre de Recherche et de Formation sur les Plantes Médicinales (CERFOPLAM) de l'Université de Lomé-Togo et au Centre de 
Recherche Biomoléculaire Pietro Annigoni (CERBA) de Ouagadougou, Burkina-Faso.

\subsection{Matériel végétal}

Les tiges feuilles de Senna occidentalis sont récoltées à Sindomé situé au nord-est de Lomé, un spécimen a été déposé à l’herbarium du Département de Botanique de la Faculté des Sciences de l'Université de Lomé sous le numéro TOGO15443. Localement cette plante est appelée Bessissan, Dankayè ou faux Kinkeliba en Ewe ou Mina.

\subsection{Préparation des extraits}

Les tiges feuillées récoltées ont été lavées et séchées à l'abri de la poussière, sous climatisation pendant 14 jours avant d'être réduites en poudre fine à l'aide d'un moulin (Thomas Scientic Laboratory Mill Model 4, USA) au Laboratoire de Physiologie et Pharmacologie de l'Université de Lomé. Deux extractions ont été effectuées à savoir l'extrait aqueux obtenu par décoction et l'extrait hydro-éthanolique 50: 50 par macération. Les différentes extractions ont été faites à raison de $100 \mathrm{~g}$ de matériel végétal pour $1000 \mathrm{~mL}$ de solvant sous agitation manuelle par intermittence pendant 72 heures. Après cela les extraits ont été filtrés avec du papier filtre puis évaporés à sec à l'aide d'un évaporateur rotatif (BUCHI, Suisse) sous vide à $40^{\circ} \mathrm{C}$. Les solvants utilisés sont l'eau distillée et l'éthanol $95^{\circ}$. Le rendement d'extraction (R) a été calculé par la formule :

$$
\mathrm{R}=(\mathrm{Me} / \mathrm{Mm}) * 100 \quad \mathrm{Mm}=\text { Masse de matière sèche }(\text { Poudre })
$$

2.4 Réalisation des cultures de Plasmodium et évaluation de l'activité antiplasmodiale in vitro des extraits.

\subsubsection{Réalisation des cultures du Plasmodium}

\subsubsection{Isolement du Plasmodium falciparum}

Le Plasmodium utilisé pour la culture est obtenu à partir du sang prélevé chez des personnes atteintes du paludisme à l'hôpital Saint Camille de Ouagadougou. Le sang a été pris dans un tube EDTA avant toute prise d'antipaludiques et après consentement du patient. Les densités parasitaires comprises entre 2000 et 80000 Plasmodium /microlitre de sang sont retenues pour la culture.

\subsubsection{Méthode de culture}

La culture des parasites a été réalisée selon la technique de Trager et Jensen (1976). Il s'agit d'une technique de culture en continu utilisant un milieu de culture semi-synthétique. Elle consiste à mettre les hématies en 
sédimentation dans un récipient à fond plat sous un flux continu d'un mélange gazeux constitué de $92 \%$ de $\mathrm{N}_{2}, 5 \%$ de $\mathrm{CO}_{2}$ et de $3 \%$ d' $\mathrm{O}_{2}$.

Le milieu de culture est constitué du milieu RPMI 1640 contenant 25 $\mathrm{mM}$ de tampon Hepes, $4 \mathrm{mM}$ de L-glutamine et $25 \mu \mathrm{g} / \mathrm{mL}$ de gentamycine. Ce milieu a été enrichi en y adjoignant $10 \%$ de sérum fœtal bovin.

Avant la mise en culture, les hématies ont été lavées 3 fois avec le milieu RPMI 1640 afin d'éliminer les leucocytes et les plaquettes. La parasitémie a été ajustée à $2 \%$ par dilution des hématies parasitées avec des hématies saines lavées provenant de donneurs sains du groupe $\mathrm{O}$; le sang du groupe $\mathrm{O}$ a été utilisé afin de limiter les risques d'incompatibilité du système $\mathrm{ABO}$ avec les hématies en culture.

L'ensemble a été mis en suspension dans le milieu de culture et l'hématocrite a été maintenu à 5\%. La suspension a alors été distribuée dans des flasques de culture cellulaire de 25 et $75 \mathrm{~mL}$ qui ont été placées dans l'incubateur à $\mathrm{CO}_{2}$. Le milieu de culture était renouvelé quotidiennement et la parasitémie était contrôlée par réalisation de frottis colorés au Giemsa et au besoin, réajustée à $2 \%$ par ajout d'hématies saines.

\subsubsection{Synchronisation des cultures au sorbitol}

Le sang de culture est récupéré à l'aide d'une pipette stérile de $10 \mathrm{ml}$ et transféré dans un tube Falcon stérile de $15 \mathrm{ml}$, puis centrifugé à $2500 \mathrm{rpm}$ pendant $7 \mathrm{mn}$. Le surnageant est récupéré puis éliminé. Une solution stérile de sorbitol (5\%) est ajoutée à raison de 5 fois le volume du culot puis homogénéisée au Vortex. Laissée au repos pendant 5 à $10 \mathrm{mn}$, la solution est centrifugée de nouveau à $2500 \mathrm{rpm}$ pendant $7 \mathrm{mn}$. Le surnageant est de nouveau éliminé. Une lame avec une goutte de culot est préparée pour vérifier si les parasites sont de même âge. Enfin le sang est suspendu de nouveau avec du milieu de culture complet à un hématocrite de $5 \%$ puis placé dans un flasque dans les conditions de culture continue des parasites.

\subsubsection{Evaluation de l'activité antiplasmodiale in vitro des extraits \\ 2.4.2.1. Préparation des solutions de drogues}

Les solutions mères des extraits à tester ont été préparées à $10 \mathrm{mg} / \mathrm{mL}$. Pour cela, $20 \mathrm{mg}$ d'extrait ont été dissous dans $2 \mathrm{~mL}$ de diméthylsulfoxide (DMSO)/Eau v/v pour les extraits hydro-éthanoliques, et dans l'eau distillée pour les extraits aqueux. Les solutions filles ont été préparées des solutions mères par prélèvement de $10 \mu 1$ de solution mère à laquelle on ajoute $990 \mu 1$ de Milieu de culture complet (MCC). L'extrait est agité au vortex pendant au moins 15 minutes. La solution obtenue a été filtrée et le filtrat a été utilisé pour la réalisation des tests antiplasmodiques. 


\subsubsection{Méthode utilisée}

Le microtest optique

Pour la réalisation du microtest optique (Basco, 2007), nous avons préparé une suspension d'hématies à $10 \%$ d'hématocrite et $5 \%$ de parasitémie, que nous avons réparti dans une plaque à 96 puits à raison de $200 \mu \mathrm{L}$ par puits. $100 \mu \mathrm{L}$ d'extrait à $50 \mu \mathrm{g} / \mathrm{mL}$ dans le DMSO ont été ajoutés aux trois premiers puits et après homogénéisation, une dilution en cascade a été effectuée à partir des premiers puits jusqu'au cinquième puits $(3,375 \mu \mathrm{g} / \mathrm{mL})$. Dans les puits témoins, les $100 \mu \mathrm{L}$ d'extrait ont été remplacés par $100 \mu \mathrm{L}$ de milieu de culture. Des puits blancs sont réalisés en remplaçant les $100 \mu \mathrm{L}$ d'extrait par $100 \mu \mathrm{L}$ d'un mélange DMSO-milieu de culture dans les mêmes proportions que dans les puits tests et la quinine est utilisée comme drogue de référence. La plaque a été recouverte et placée dans l'incubateur à $\mathrm{CO}_{2}$ pendant 72 heures. Le milieu de chaque puits était renouvelé toutes les 24 heures et à chaque occasion des frottis sanguin sont été réalisés à partir de chacun des puits; après fixation au méthanol et coloration au Giemsa 10\% pendant 15 minutes, la parasitémie de chaque puits a été déterminée afin d'évaluer le pourcentage d'inhibition de la croissance du parasite.

\section{Calculs des pourcentages d'inhibition}

Les résultats obtenus à partir de ces tests sont été exprimés en pourcentage d'hématies parasitées. Les moyennes des trois puits de chaque dilution ainsi que des puits blancs ont été calculées. Les moyennes des puits témoins ont également été calculées et représentent $100 \%$ de croissance soit $0 \%$ d'inhibition. Les valeurs des pourcentages d'inhibition ont été déterminées à partir de la formule suivante:

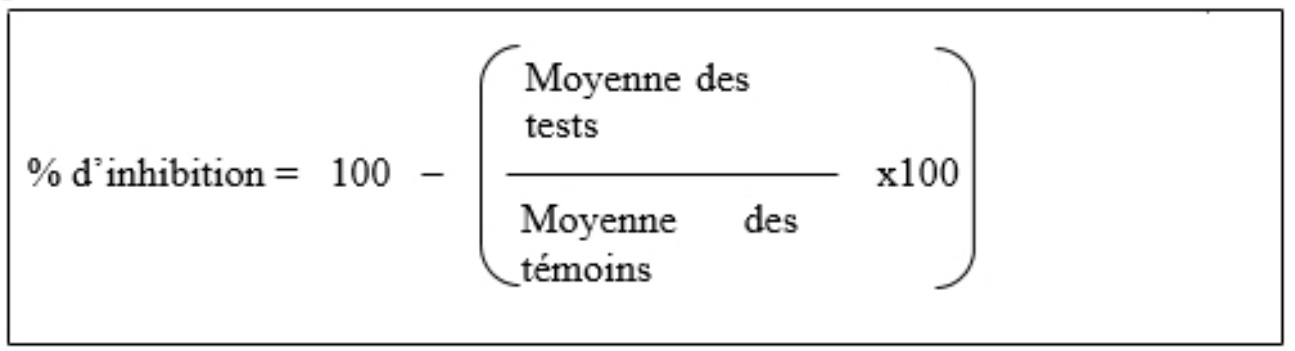

Le rapport de réduction parasitaire (RRP) a également été évalué pour chaque extrait en appliquant la formule suivante:

$\mathrm{RRP}=\frac{\text { Densité parasitaire initiale J0 }}{\text { Densité parasitaire du test }}$


Ce rapport est directement proportionnel à l'activité antiplasmodique de l'extrait. Plus il est élevé, plus l'extrait est actif.

\subsubsection{Traitement des données}

Les résultats sont exprimés sous forme de moyenne \pm ESM. Les logiciels Graph Pad Prism 5.0 du Table Curve 2Dv5 et Microsoft office Excel 2007 et Excel 2013 ont été utilisés pour le traitement des données, le tracé des courbes et le calcul des $\mathrm{CL}_{50}$ et $\mathrm{CI}_{50}$ des extraits testés.

\subsection{Evaluation de l'activité antioxydante}

\subsubsection{Evaluation des phénols totaux}

L'évaluation de la concentration en polyphénols des différents extraits a été réalisée selon la méthode colorimétrique décrite par Al-Farsi et al. (2005) avec quelques modifications. Cent microlitres d'extrait à la concentration de $1 \mathrm{mg} / \mathrm{mL}$ ont été mélangés à $750 \mu \mathrm{L}$ de réactif de Folin-Ciocalteu dilué au $1 / 10$ ème. Après $5 \mathrm{mn}$ d'incubation à $25^{\circ} \mathrm{C}, 750 \mu \mathrm{L}$ d'une solution de carbonate de sodium (Na2CO3) à $20 \%$ y ont été ajoutés. Le mélange obtenu a été incubé pendant $90 \mathrm{mn}$ à l'abri de la lumière puis la densité optique de chaque mélange a été mesurée à $\lambda=765 \mathrm{~nm}$ contre le blanc. L'acide gallique à différentes concentrations $(50-500 \mu \mathrm{g} / \mathrm{mL})$ a servi de standard. La concentration en polyphénols est déterminée par extrapolation de la courbe de calibration obtenue à partir des différentes concentrations d'acide gallique dont l'équation est : $\mathrm{y}=10,2 \mathrm{x}-0,039$.

\subsubsection{Test de réduction du radical libre DPPH}

La mesure de l'activité antiradicalaire des différents extraits a été effectuée selon la méthode décrite par McCune et Johns (2002). La solution de DPPH a été fraîchement préparée dans du méthanol $(100 \mu \mathrm{mol} / \mathrm{L})$. Une gamme de concentrations de chaque extrait $(0-100 \mu \mathrm{g} / \mathrm{mL})$ a été également préparée dans du méthanol. Brièvement, un volume de $0,25 \mathrm{~mL}$ des solutions de chaque extrait ou du standard (Quercétine) a été ajouté à 1,5 mL de la solution de DPPH. Le mélange a été vigoureusement homogénéisé puis incubé à la température ambiante $\left(25^{\circ} \mathrm{C}\right)$. Après $30 \mathrm{mn}$ d'incubation à l'obscurité, l'absorbance de chaque concentration des extraits étudiés a été mesurée à la longueur d'onde $\lambda=517 \mathrm{~nm}$ à l'aide du spectrophotomètre UV-visible (Genesys 10S UV-Vis Spectrophotometer, USA). La quercétine est utilisée pour générer une courbe standard afin de déterminer la concentration inhibitrice $50 \%\left(\mathrm{CI}_{50}\right)$ des extraits. Le pourcentage d'inhibition du radical DPPH a été calculé par l'équation suivante :

$\%$ d'inhibition $=(\mathrm{A} 0-\mathrm{AE} / \mathrm{A} 0) \times 100$

Où $\mathrm{A} 0$ est l'absorbance du blanc réactif, et $\mathrm{AE}$ est l'absorbance des extraits testés. Les essais ont été réalisés en triplicata. 
2.6. Etude de la toxicité des extraits sur les larves de crevettes (Artemia salina)

\subsubsection{Principe}

Ce test est basé sur la survie des larves de crevettes dans l'eau de mer en présence de la solution à tester. En effet, il existe une corrélation entre cytotoxicité sur les larves de crevettes et sur les cellules 9PS et 9KB (carcinome nasopharyngien humain) d'une part (Pelka et al., 2000), les cellules A-549 du carcinome pulmonaire et les cellules HT-29 du carcinome du côlon d'autre part (Carballo et al., 2002). Ce test est très utile pour établir la toxicité des extraits de plantes et a été largement employé dans ce sens (Sam, 1993).

\subsubsection{Description du test}

Les œufs d'Artemia salina sont incubés dans l'eau de mer sous agitation jusqu'à éclosion de jeunes larves (48 heures). Une série de solutions des substances (extraits) est préparé à des concentrations variables et progressives. Un nombre défini de larves est introduit dans chaque solution. Toutes les solutions ainsi que des solutions témoins ne contenant pas de substance active sont laissées sous agitation pendant 24 heures. Le comptage sous microscope du nombre de larves survivantes dans chaque solution permet d'évaluer la toxicité de la solution. Dans le cas où des décès sont constatés dans le milieu témoin, les données sont corrigées par la formule de Abbott : \% décès $=[$ (test - témoin $) /$ témoin $)] \times 100$. Pour ce travail une colonie de 16 larves a été utilisée dans chaque solution. Les données dose-réponse sont transformées par logarithme et la $\mathrm{CL}_{50}$ est ainsi déterminée par une régression linéaire (Houngbeme et al., 2014).

\subsubsection{Préparation des solutions à tester}

Dans cette étude, les extraits aqueux et hydro-éthanoliques ont été préparés à raison de $50 \mathrm{mg} / \mathrm{mL}$ de solvant de dissolution. Nous avons procédé ensuite à dix (10) dilutions successives au demi (1/2) de la solution stock avec l'eau de mer. Les concentrations exprimées en $\mathrm{mg} / \mathrm{mL}$ des solutions diluées contenues dans des tubes à essais numérotés de 1 à 10 ont été respectivement de 50/2;50/4;50/8;50/16;50/32;50/64;50/128;50/256;50/512 et $50 / 1024$. Les tests ont été répétés trois fois pour assurer la fidélité des résultats obtenus.

\subsection{Screening phytochimique}

\subsubsection{Chromatographie sur couche mince (CCM)}

Les différents extraits ont été dissous à $100 \mathrm{mg} / \mathrm{mL}$ dans leurs solvants d'extraction. A l'aide de tubes capillaires, les solutions d'extraits ont été déposées sur une chromatoplaque, de même que les témoins de référence. La 
plaque a été séchée à l'air ambiant et a ensuite été placée dans une cuve de migration contenant le système de solvants approprié. Après la migration, la plaque a été retirée et séchée, puis la révélation a été faite à l'aide d'un réactif spécifique pour la détection des groupes chimiques.

Après la révélation, le rapport frontal (Rf) de chaque spot a été calculé. Il s'agit du rapport : distance parcourue par le soluté / distance parcourue par le solvant. Ce rapport permet de caractériser les composés séparés (Ouédraogo, 2011).

\subsubsection{Screening des groupes chimiques}

Le screening phytochimique a été réalisé selon la méthode de Houghton et Raman (1998) à la recherche des alcaloïdes, des composés polyphénoliques (les tanins, des flavonoïdes, des anthocyanes, des leucoanthocyanes), des dérivés quinoniques, des saponosides, des triterpénoïdes et de stéroïdes, des dérivés cyanogéniques, des mucilages, des coumarines, des composés réducteurs, des dérivés anthracéniques, des O-hétérosides,des Chétérosides et des hétérosides cardiotoniques.

\section{Résultats}

\subsection{Test antiplasmodial}

La courbe montrant l'activité antiplasmodiale de la quinine (Figure 1) est obtenue par l'équation: $\mathrm{y}=3,19 \mathrm{x} / \mathrm{lnx}-048 \mathrm{x}+79,60$ avec $\mathrm{r}^{2}=0,9394(\mathrm{p}$ $=0,0008$ ) avec une $\mathrm{CI}_{50}=0,45 \pm 0,01 \mu \mathrm{g} / \mathrm{mL}$. Les résultats montrent que l'extrait aqueux a une activité modérée sur le $P$. falciparum avec une $\mathrm{CI}_{50}$ de $91,08 \pm 0,61 \mu \mathrm{g} / \mathrm{mL}$ (Figure 2) dont l'équation de la courbe d'inhibition est $\mathrm{y}=3,77 \mathrm{e}^{\mathrm{x}}+57,17 \ln \mathrm{x}-265,70$ avec $\mathrm{r}^{2}=0,99$ tandis que l'extrait hydroéthanolique est très actif avec une $\mathrm{CI}_{50}$ de $1 \pm 0,05 \mu \mathrm{g} / \mathrm{mL}$ (Figure 3) avec $\mathrm{r}^{2}$ $=0,99$ et $\mathrm{y}=655.70 \ln \mathrm{x} / \mathrm{x}^{2}-7.10^{-4} \mathrm{x}^{2,5}-280.47 / \mathrm{x}^{0,5}+149,09$.

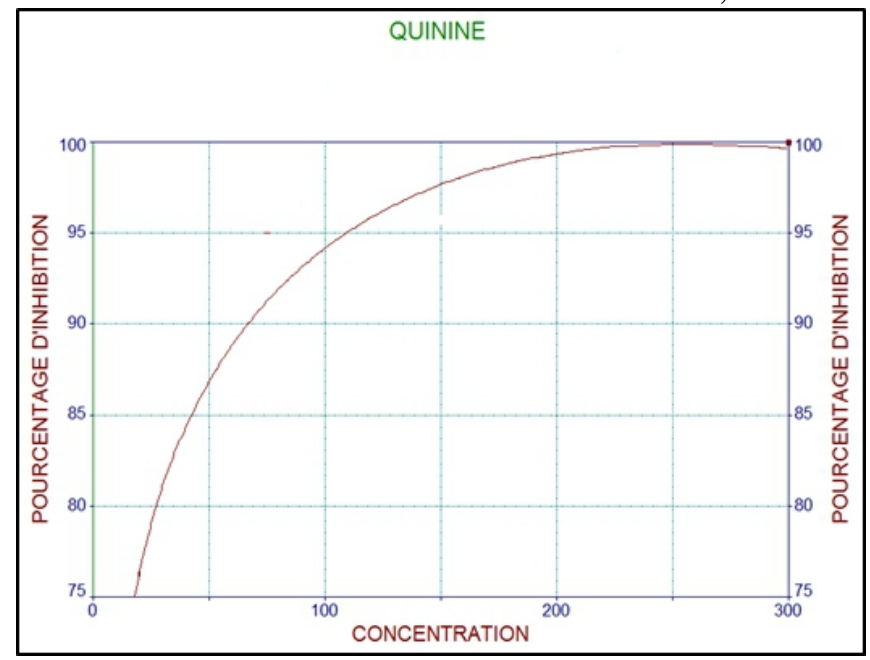

Figure 1 : Activité antiplasmodique de la quinine. 


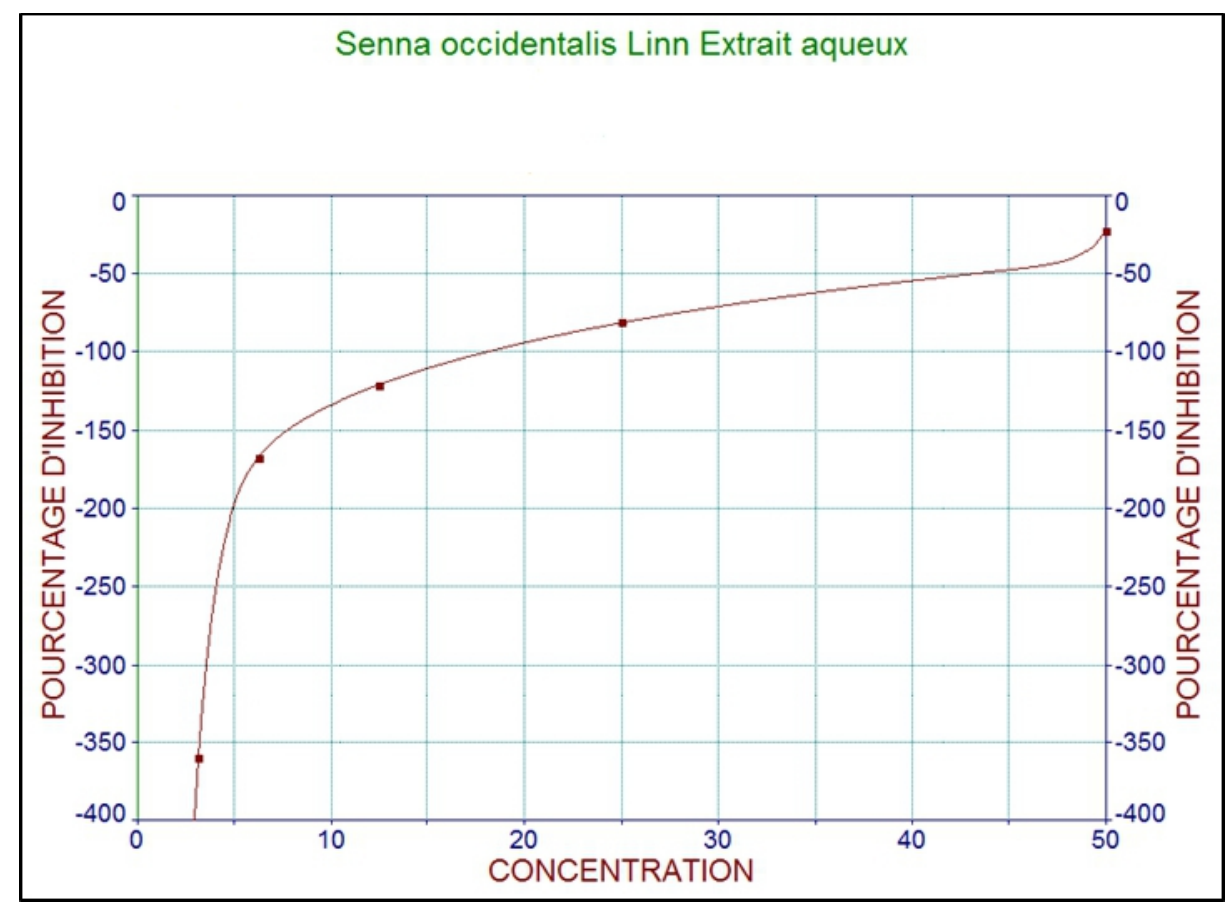

Figure 2: Activité antiplasmodique de l'extrait aqueux de Senna occidentalis.

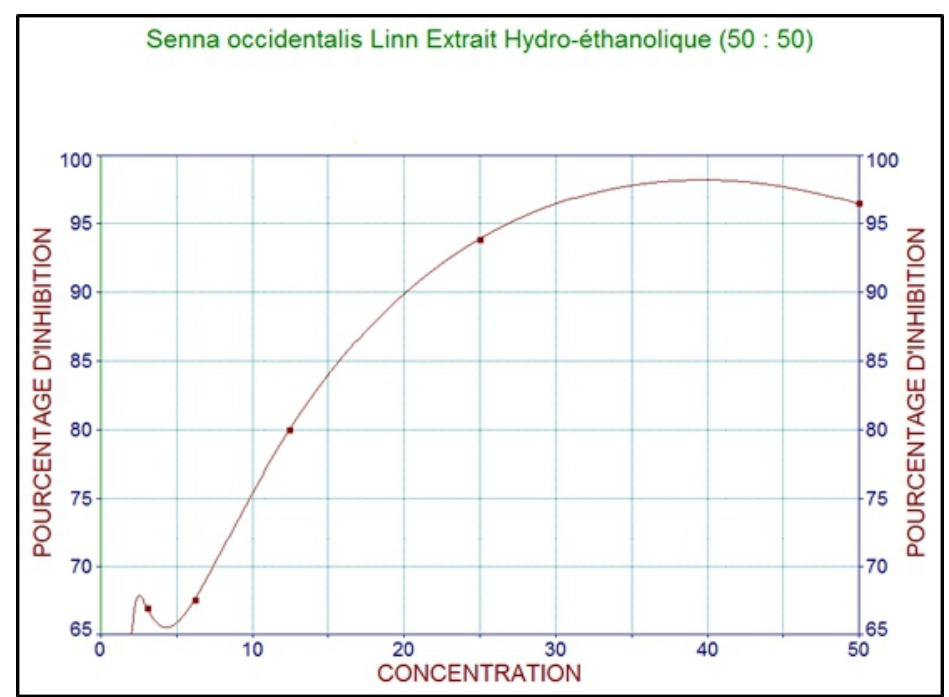

Figure 3 : Activité antiplasmodique de l'extrait hydro-éthanolique de Senna occidentalis.

\subsection{Test antiradicalaire}

$\mathrm{Au}$ niveau de l'activité antiradicalaire les résultats sont enregistrés dans le tableau 1.

Selon les tests du DPPH, Les CI50 des différents extraits du Senna occidentalis sont très élevées tandis que celui du quercétol a une valeur basse. 
Les valeurs de polyphénols obtenues ne sont pas élevées, cela montre que Senna occidentalis est moins riche en polyphénols.

Tableau 1 : Activité antioxydante in vitro des extraits

\begin{tabular}{|l|c|c|c|c|}
\hline Plantes et recettes de plante & \multicolumn{2}{|c|}{$\mathrm{DPPH}\left(\mathrm{CI}_{50}, \mu \mathrm{g} / \mathrm{mL}\right.$ d'extrait $)$} & \multicolumn{2}{c|}{ Polyphénols (mgEAG/g d'extrait) } \\
\cline { 2 - 5 } & $\begin{array}{c}\text { Extraits } \\
\text { Hydro-éthanolique }\end{array}$ & Extraits aqueux & $\begin{array}{c}\text { Extraits } \\
\text { Hydro-éthanolique }\end{array}$ & Extraits aqueux \\
\hline Senna occidentalis Linn & $572,49 \pm 0,01$ & $1552,01 \pm 0,01$ & $44,30 \pm 8,11$ & $25,27 \pm 10,00$ \\
\hline Quercétol & \multicolumn{2}{|c|}{$2,05 \pm 0,04$} & - & - \\
\hline
\end{tabular}

Les valeurs sont exprimées en Moyenne \pm ESM. EAG : Equivalent d'acide gallique.

\subsection{Test toxicologique}

Le pourcentage de mortalité des larves est déterminé après une exposition de 24 heures aux extraits testés, rapportée à la mortalité moyenne à 10 concentrations entre 0,024 à $12,5 \mathrm{mg} / \mathrm{mL}$. Aucune mortalité n'a été observée dans la solution de solubilisation des extraits (eau de mer). A partir de ces courbes sont extraites par régression logarithmique linéaire les valeurs de CL50 des différents extraits testés (Tableau 2).

Tableau 2: Concentration létale 50\% des larves d'Artemia salina de même âge.

\begin{tabular}{cll}
\hline & CL50 en $\mathrm{mg} / \mathrm{mL}$ & \\
Plante & Extrait Hydro-éthanolique $(50: 50)$ & Extrait aqueux \\
\hline Senna occidentalis Linn & 3,31 & 3,70
\end{tabular}

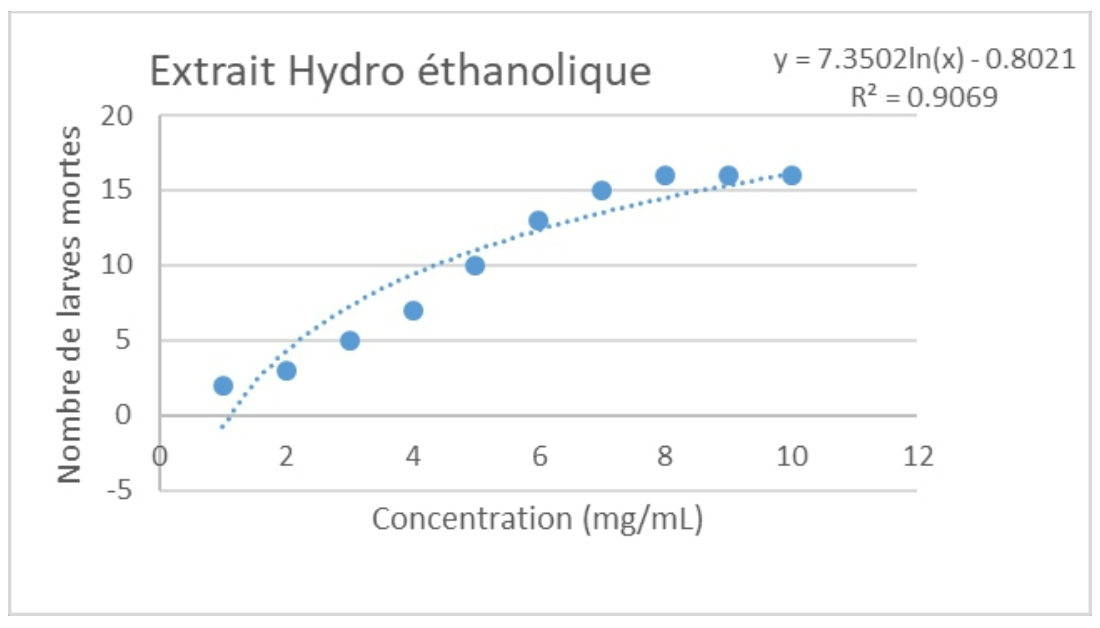




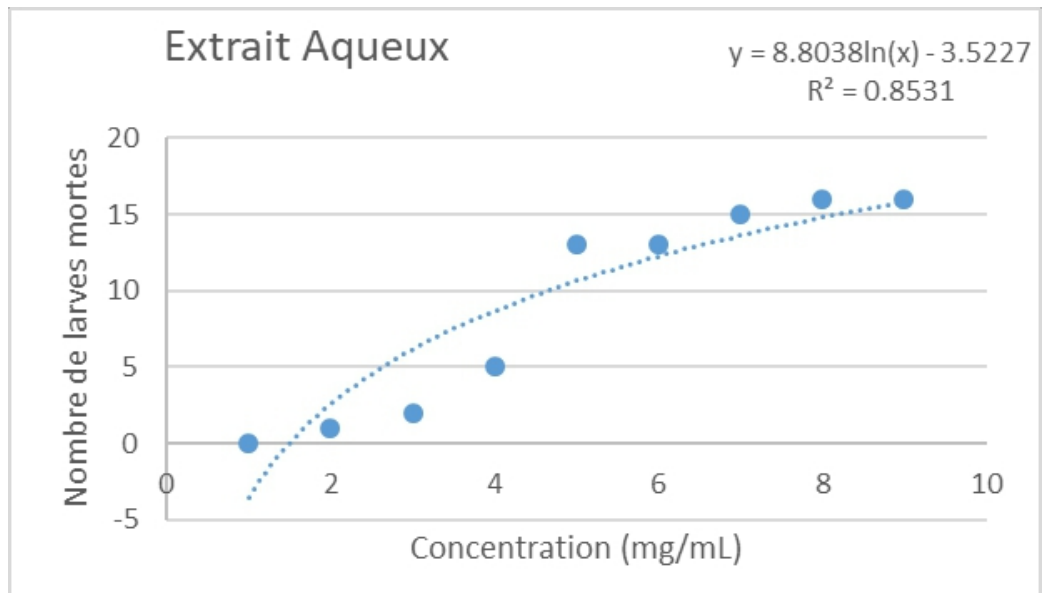

Figure 4 : Toxicité larvaire de l'extrait hydro éthanolique (A) et aqueux (B) de Senna occidentalis.

\subsection{Screening phytochimique}

La chromatographie sur couche mince $(\mathrm{CCM})$ a révélé la présence de Flavonoïdes, Tanins, Saponosides, Coumarines et des Alcaloïdes dans les différents extraits. Les résultats du test colorimétrique sont résumés dans le tableau 3.

Tableau 3: Tests qualitatifs des composés chimiques

\begin{tabular}{|l|cccccccccccccccccc}
\hline Composés chimiques & Al & TACTAG & Fl & Ant & Leu & Qn & SP & Tp & St & Cy & Mu & Cm & Cr & HL & O-H C-H H-C \\
\hline Senna occidentalis Linn & + & + & + & + & ++ & - & + & + & - & - & - & ++ & + & - & - & - & - & - \\
\hline
\end{tabular}

TAG : Tanins galliques ; TAC: Tanins catechiques ; Al: Alcalofdes ; Fl: Flavonoides ; St:

Steroides ; Tp : terpenes ; Leu :Leucoanthocyanes ; Ant: Anthocyanes ; Mu : Mucilages ; O-H: O-Heterosides ; C-H: C-Heterosides ; HL: Heterosides libres ; Cr: Composes reducteurs ; $\mathbf{C m}$ : Coumarines ; Hc : Heterosides cardiotoniques ; Qn : Quinones ; Sp : Saponosides ; Cy: derives cyanogeniques.

\section{Discussion}

L'extrait aqueux présente un rendement plus élevé que celui de l'extrait hydro-éthanolique. Ce même constat a été fait par Weniger et al., (2004) ; Tona et al., (1999 ; 2004) et que pour un même organe de plante, avec différents solvant d'extraction, les extraits les plus efficaces sur le $P$. falciparum in vitro sont ceux obtenus avec un faible rendement (Okpekon, 2006 ; Mbatchi et al., 2006 ; Koudouvo, 2009). La même constatation a été faite dans cette étude. L'activité antiplasmodiale observée au niveau du Senna occidentalis justifie effectivement l'utilisation traditionnelle de cette espèce dans le traitement du paludisme. Les extraits : éthanolique, éther de pétrole de la feuille de Senna occidentalis ont donné respectivement des $\mathrm{CI}_{50}$ de $5,3 \mu \mathrm{g} / \mathrm{mL}$ et $1,4 \mu \mathrm{g} / \mathrm{mL}$ (Tona et al., 2004) ; pour le même extrait éthanolique des feuilles, Zirihi et al., (2005) et Nassirou et al., (2015) ont obtenu 
respectivement des $\mathrm{CI}_{50}$ de $36, \pm 5,6 \mu \mathrm{g} / \mathrm{mL}$ et $10,4 \mu \mathrm{g} / \mathrm{mL}$ puis Ménan et al., (2006) ont trouvé que l'extrait aqueux n'est pas actif comparativement à l'extrait éthanolique et pentanolique. Ces résultats sont semblables à ceux obtenus par nos travaux. Par contre, l'extrait méthanolique des racines de la plante n'est pas antiplasmodique car la $\mathrm{CI}_{50}$ est supérieure à $100 \mu \mathrm{g} / \mathrm{mL}$ (Addae-Kyereme et al., 2001). L'utilisation de l'extrait hydro-éthanolique de la feuille du Senna occidentalis indique une $\mathrm{CI}_{50}$ de $45 \mu \mathrm{g} / \mathrm{mL}$ sur une souche chloroquino-résistante W2 strain du Plasmodium falciparum (Kaou et al., 2008). De ces résultats, les tiges feuillées produiraient donc plus d'action sur le Plasmodium falciparum que les feuilles. D'autres espèces de la même famille ont prouvé aussi leur activité antiplasmodique telle que Cassia angustifolia qui a eu une $\mathrm{CI}_{50}$ de $37,5 \mu \mathrm{g} / \mathrm{mL}$ (Somayeh et al., 2015). Senna occidentalis est par ailleurs utilisé d'après la littérature comme plante intervenant dans le traitement de plusieurs pathologies telles que : la fièvre, les maux de ventre, les gastralgies, les blennorragies, purgatif, les hémorroïdes, les anémies, cette plante possède effectivement une activité antiplasmodiale. Cette plante n'est pas antioxydante car elle présente une $\mathrm{CI}_{50}$ plus élevée que celle du quercétol. Par ailleurs Senna occidentalis est pauvre en polyphénol. Ces résultats montrent que plus un extrait est riche en polyphénol, plus ce dernier est antioxydant car les polyphénols ou composés phénoliques sont des molécules spécifiques du règne végétal. Donc les extraits du Senna occidentalis agiraient par stress oxydatif. La lecture des courbes (figure 4) montre que les larves sont sensibles aux extraits selon une relation de dose-réponse car le nombre de larves mortes est proportionnel à la concentration et ceci au niveau de chaque extrait (Fagbohoun, 2014). De même, les coefficients de corrélations $\left(\mathrm{r}^{2}\right)$ sont de 0,85 et 0,90 donc supérieurs ou très voisins de 0,8 ; on en déduit qu'il y a une bonne corrélation entre les concentrations testées et les différentes réponses enregistrées. Selon les CL50 de l'extrait hydro-éthanolique et de l'extrait aqueux Senna occidentalis ne présentent pas de toxicité à la dose appliquée car les CL50 obtenues sont largement supérieures à $0,1 \mathrm{mg} / \mathrm{mL}$ (Mousseux, 1995). Cependant, en considérant la corrélation entre la cytotoxicité sur les larves de crevettes et sur les cellules 9PS et 9KB (carcinome nasopharyngien humain) d'une part (Pelka et al., 2000), les cellules A-549 du carcinome pulmonaire et les cellules HT29 du colon d'autre part (Carballo et al., 2002), on peut dire sous réserve de poursuivre les investigations, que les extraits testés ne présentent pas d'activité cytotoxique et peuvent donc être utilisées sans aucun risque de toxicité à court et moyen terme. Donc ce test de toxicité larvaire ("brine shrimp") constitue alors un screening préliminaire pour déterminer non seulement le degré de toxicité d'un produit, mais aussi la présence de potentiels composés anticancéreux (Meyer et al., 1982). En effet, lors d'une évaluation toxicologique d'extraits de plantes par ce test de toxicité, une valeur de CL50 
$<1000 \mathrm{~g} / \mathrm{mL}$ est considérée comme bioactive (Meyer et al., 1982). Il est un dépistage préliminaire de la toxicité des extraits végétaux (Ghosh et al., 2015; Kibiti et Afolayan, 2016; Oberlies et al., 1998; Sufian et Haque, 2015; Syahmi et al., 2010), toxines fongiques (Harwing et Scott., 1971), métaux lourds (Saliba et Krzyz, 1976), toxines cyanobactériennes (Hisem et al., 2011), pesticides (Michael et al., 1956), tests de cytotoxicité sur des matériaux dentaires (Pelka et al., 2000) et nanostructures (Maurer-Jones et al., 2013). Pour le composé bioactif d'origine naturelle ou synthétique, il s'agit d'un test rapide et complet (Quazi et al., 2017). Tous ces résultats sont obtenus à cause de la composition phytochimique du Senna occidentalis car ce caractère non toxique de ces extraits est observé suite à l'absence dans ces échantillons, des hétérosides cardiotoniques et des dérivés cyanogéniques qui sont généralement des composes toxiques (Houngbeme et al., 2014). Par ailleurs, la littérature informe que les grands groupes chimiques sont à la base de l'expression de l'activité antiplasmodiale d'une plante car selon certains auteurs, chacune des familles de métabolites secondaires peut potentiellement abriter des molécules à activité antiplasmodiale : les tanins, les flavonoïdes et les saponines (Banzouzi et al., 2002; Abdoulaye et al., 2009 ; Ramazani et al., 2010 ; Ravikumar et al., 2011a ; Garcia-Alvarez et al., 2015), les quinones (Obodozie et al., 2004 ; Ayo et al., 2010), les triterpènes et stéroïdes (Camacho et al., 2000 ; Wright et al., 2002 ; Moon et al., 2007 ; Ravikumar et al., 2011) ; les alcaloïdes (Wright et al., 1991 et 2001 ; Ancolio et al., 2002 ; Sanon et al., 2003a et 2003b ; Bonjean et al., 1998 ; Oliveira et al., 2009) ; les phénols (Ravikumar et al., 2011a); les glycosides (Ene et al., 2009) ; les protéines (Ravikumar et al., 2011b) et les résines (Ravikumar et al., 2011c). $\mathrm{Au}$ Niger les travaux de Nassirou et al en 2015 ont montré que l'activité antiplasmodiale $\left(\mathrm{CI}_{50}=10,04 \mu \mathrm{g} / \mathrm{mL}\right)$ observée au niveau de Senna occidentalis proviendrait de sa moins riche composition en polyphénols, quinones, triterpènes et stéroïdes tandis que les travaux de Tona et al., (2004) ont obtenu par contre, une très forte activité antiplasmodiale $\left(\mathrm{CI}_{50}=2,8 \pm 0,5\right.$ $\mu \mathrm{g} / \mathrm{mL}$ ) de l'extrait éthanolique des feuilles de Senna occidentalis et que cette activité serait due à la présence de certaines molécules du groupe des quinones, des flavonoïdes, des triterpènes et des stéroïdes.

\section{Conclusion}

L'extrait hydroéthanolique de la tige feuillée de Senna occidentalis possède effectivement une efficacité sur le Plasmodium falciparum. Cette plante n'étant pas cytotoxique donc peut valablement être conseillée dans le traitement du paludisme. Mais la réalisation d'autres tests pharmacologiques et toxicologiques seraient nécessaires pour rassurer son innocuité. 


\section{References:}

1. Abdoulaye A., Moussa I., Ousmane A., Ikhiri K. (2009). Rapport d'Activité de Recherche sur les Plantes Antipaludiques du Laboratoire de Chimie des Substances Naturelles, Département de Chimie/FAST/UAM Niger.

2. Addae-Kyereme J., Simon C., Howard K., Colin W. W. (2001). Antiplasmodial activities of some Ghanaian plants traditionally used for fever/malaria treatment and of some alkaloids isolated from Pleiocarpa mutica; in vivo antimalarial activity of pleiocarpine. Journal of Ethnopharmacology: 99-103. ·

3. Agbodeka, K., Gbekley, E. H., Karou S. D., Anani K., Simpore, J. (2017a). Activité antiplasmodiale des plantes médicinales d'Afrique de l'Ouest: Revue de la littérature [Antimalarial activity of medicinal plants from West Africa: A review]. International Journal of Innovation and Scientific Research. ISSN 2351-8014 Vol. 28 No. 2 Jan. 2017, pp. 121-129.

4. Al-Farsi M., Alasalvar C., Morris A., Barron M., Shahidi F. (2005). Comparison of antioxidant activity, anthocyanins, carotenoïds, and phenolics of three native fresh and sun-dried date (Phoenix dactylifera L.). Journal of Agricultural and Food Chemistry: 7592-7599.

5. Ancolio C., Azas N., Mahiou V., Ollivier E., Di Giorgio C., Keita A., Timon-David P., Balansard G. (2002). Antimalarial activity of extracts and alkaloids isolated from six plants used in traditional medicine in Mali and Sao Tome. Phytotherapy Research. : 646-9.

6. Ayo R. G. (2010). Phytochemical constituents and bioactivities of the extracts of Cassia nigricans Vahl: A review. Journal of Medicinal Plants Research: (14), pp. 1339-1348.

7. Banzouzi J.T., Prado R., Mena H., Valentin A., Roumetan C., Mallie M., Pelissier Y., Blache Y. (2002). In vitro antiplasmodial activity of extracts of Alchornea cordifolia and identification of an active constituent: ellagic acid. Journal of Ethnopharmacology: 81, 399401.

8. Bonjean K., De Pauw-Gillet M.C., Defresne M.P., Colson P., Houssier C., Dassonneville L., Bailly C., Greimers R., Wright C., QuetinLeclercq J., Tits M., Angenot L. (1998). The DNA intercalating alkaloid cryptolepine interferes with topoisomerase II and inhibits primarily DNA synthesis in B16 melanoma cells. Biochemistry: 37, 5136-5146.

9. Camacho C.R., Croft S.L., Phillipson J.D. (2000). Natural products as sources of antiprotozoal drugs. Current Opinion in Anti-Infective Investigational Drugs: 2, 47-62. 
10. Carballo J.L., Hernandez-Inda Z.L., Perez P., Garcia-Gravalos M.D. (2002). Comparaison between to brine shrimp assays to detect in-vitro cytotoxicity in marine natural products, BMC. Biotechnology :17.

11. Cilundika M. P., Nyota N. O., Numbi O. L. (2016). Problématique de l'utilisation des Moustiquaires Imprégnées d'insecticide à Longue Durée (MILD) chez les enfants de moins de 5 ans en République Démocratique du Congo. The panafrican medical journal: 101.

12. Courcoux G. (2008). Dossier de presse de l'Institut de Recherche en Développement (IRD). Journée mondiale de lutte contre le paludisme : $25 p$.

13. Dénou A. (2011). Enquête ethnobotanique et étude des propriétés analgésiques des plantes antipaludiques du Togo et du Mali. Mémoire de DEA en Biologie de Développement, Option: Physiologie Pharmacologie, Faculté des Sciences, Université de Lomé, 120 p.

14. Ene AC, Atawodi SE, Ameh DA, Kwanashie HO, Agomo PU. (2009). In vivo antiplasmodial effect of chloroform extracts of Artemisia maciverae Linn and Artemisia maritima Linn. African Journal of Biotechnology: 6612-6616.

15. Esseh K. (2012). Enquête ethnobotanique sur les plantes traitant le paludisme grave au sud Togo. Mémoire de DEA en Biologie de Développement, Option: Physiologie Pharmacologie, Faculté des Sciences, Université de Lomé, 89 p.

16. Fagbohoun L. (2014). Identification et évaluation de l'activité inhibitrice des polyphénols des folioles de palmier à huile (Elaeis guineensis Jacq) du site de Pobè sur le développement des larves de Coelaenomenodera lameensis Berti contaminant ces palmiers. Mémoire de DEA en Chimie Université d'Abomey-Calavi, 80 p.

17. García-Álvarez A., Casper H.A.V.L., Carlos J.L., Hussner A., VélezMartín A., Pérez-Vázquez A., Andy J.G., Castellanos E.M. (2015). Internal transport of alien and native plants by geese and ducks - an experimental study. Freshwater Biology: doi:10.1111/fwb.12567.

18. Ghosh A., Banik S., Islam M. (2015). In vitro thrombolytic, anthelmintic, anti-oxidant and cytotoxic activity with phytochemical screening of methanolic extract of Xanthium indicum leaves. Bangladesh Journal of Pharmacology: 854-59.

19. Harwing J., Scott P. (1971). Brine shrimp (Artemia salina L.) larvae as a Screening system for fungal toxins. Journal of Applied Microbiology: 1011-1016.

20. Hisem D., Hrouzek P., Tomek P., Tomšičková J., Zapomělová E., Skácelová K., Lukešová A., Kopecký J. (2011). Cyanobacterial cytotoxicity versus toxicity to brine shrimp Artemia salina. Toxicon Journal: 76-83. 
21. Houghton P.J., Raman A. (1998). Laboratry Handbook for the Fractionation of Naturals Extracts. Editions Chapman and Hall first collection, New York, NY USA.

22. Houngbeme A.G., Gandonou C., Yehouenou B., Kpoviessi S.D.S., Sohounhloue D., Moudachirou M., Gbaguidi FA. (2014). Phytochemical analysis, toxicity and antibacterial activity of Benin medicinal plants extracts used in the treatment of sexually transmitted infections associated with HIV/AIDS. International Journal of Pharmaceutical Sciences Research: 1739-1745.

23. Kaou A.M., Mahiou-Leddet V., Hutter S., Aïnouddine S., Hassani S., Yahaya I., Azas N., Ollivier E. (2008). Antimalarial activity of crude extracts from nine African medicinal plants. Journal of Ethnopharmacology: 74-83.

24. Kibiti C., Afolayan A. (2016). Antifungal activity and brine shrimp toxicity assessment of Bulbine abyssinica used in the folk medicine in the Eastern Cape Province, South Africa. Bangladesh Journal of Pharmacology : 469-77.

25. Koudouvo K. (2006). «Etude botanique et ethnobotanique des plantes médicinales antipaludiques de la Région Maritime du Togo ». Mémoire de DEA, Biologie de Développement. Faculté des Sciences, Université de Lomé Togo, 60 p.

26. Koudouvo, K. (2009). Contribution à la recherche sur les plantes médicinales à propriété antipaludiques du Togo. Thèse de Doctorat de l'Université de Lomé en Biologie de Développement. Option: Ethnobotanique et Pharmacologie des Substances Naturelles, 182p.

27. Maurer-Jones M.A., Love S.A., Meierhofer S., Marquis B.J., Liu Z., Haynes C.L. (2013). Toxicity of nanoparticles to brine shrimp: An introduction to nanotoxicity and interdisciplinary science. Journal of Chemical Education: 475-78.

28. Mbatchi S.F., Mbatchi B., Banzouzi- Bansimba J.T., Nsonde N.G.F., Oumba J.M., Berry A., Benoit-Vical F. (2006). In vitro antiplasmodial activity of 18 plants used in Congo Brazzaville traditional Medicine. Journal of ethnopharmacology: 168-174.

29. McCune L.M., Johns T. (2002). Antioxidant activity in medicinal plants associated with the symptoms of diabetes mellitus used by indigenous peoples of North American boreal forest. Journal of Ethnopharmacology: 197-205.

30. Ménan H., Banzouzi J.T., Hocquette A., Pélissier Y., Blache Y., Koné M., Mallié M., Assi LA., Valentin A. (2006). Antiplasmodial activity and cytotoxicity of plants used in West African traditional medicine for the treatment of malaria. Journal of Ethnopharmacology: 131-6. 
31. Meyer B.N., Ferrign R.N., Putnam J.E., Jacobson L.B., Nicholas D.E., McLaughlin J.L. (1982). Brine shrimp: a convenient general bioassay for active plant constituents. Planta Medica: 31-34.

32. Moon H.I., Jung J.C., Lee J. (2007). Antiplasmodial activity of triterpenoids isolated from whole plants of Viola genus from South Korea. Parasitology Research: 641-644.

33. Nassirou R.S., Sadou R., Maman L.I., Ilagouma A.T., Mahamadou A. (2015). Évaluation in vitro de l'activité antiplasmodiale d'extraits de plantes issues de la pharmacopée traditionnelle du Niger. Journal of Applied Biosciences: 8291-8300.

34. Oberlies N.H., Rogers L.L., Martin J.M., McLaughlin J.H. (1998). Cytotoxic and insecticidal constituents of the unripe fruit of Persea americana. Journal of Natural Product: 781-85.

35. Obodozie O.O., Okpako L.C., Tarfa F.D., Orisadipe A.T., Okogun J.I., Inyang U.S., Ajaiyeoba E.O., Wright C.W. (2004). Antiplasmodial principles from Cassia nigricans. Pharmaceutical Biology. ISSN: 1388-0209. Issue 8. Page : 626-628.

36. Okpekon T.A. (2006). Etude chimique et biologique de plantes ivoiriennes utilisées en médecine traditionelle en tant qu'antiparasitaires : Aframomum sceptrum K. Schum. (Zingiberaceae), Bridelia Ferruginea Benth. (Euphorbiaceae), Isolona cooperi Hutch. \& Dalz et Uvaria afzelii Sc. Elliot (Annonaceae). Thèse de doctorat en Phytochimie. Soutenue en 2006 à Paris 11.

37. Oliveira V.C.S., Moura D.M.S., Lopes J.A.D., D’Andrade P.P., Da Silva N.H., Figueiredo R.C.B.Q., (2009). Effects of essential oils from Cymbopogon citratus (DC) Stapf, Lippia sidoides Cham and Ocimum gratissimum L. on growth and ultrastructure of Leishmania chagasi promastigotes. Parasitology Research: 1053-1059

38. OMS. (2002, 2017): Rapport sur le paludisme dans le monde, année 2002, 2017.

39. OMS. (2010). Prévention et traitement du paludisme à Plasmodium falciparum résistant à l'artémisinine: mise à jour à l'intention des voyageurs internationaux. REH : 195-196

40. OMS. (2013). Paludisme. Available from: http://www.who.int/mediacentre/factsheets/fs094/fr/index.html accès le 27 avril 2013.

41. Ouédraogo D.F. (2011). Etude in vitro de l'activité antiplasmodique d'extraits de feuilles, de fleurs et de galles de Guiera senegalensis J.F. Gmel (COMBRETACEAE), Thèse de doctorat. Université de Ouagadougou. 105p.

42. Pelka M., Danzl C., Distler W., Petschelt A., (2000). A new screening test toxicity testing of dental materials, Journal of Dentisty: 341-345. 
43. PNLP. (2009). Evaluation de l'efficacité de «DIMILIN » sur les vecteurs du paludisme à Lomé (TOGO).

44. PNLP. (2014). Rapport annuel du programme national de lutte contre le paludisme.

45. Quazi S.S., Fatema C.A., Misbahuddin M. (2017). Brine shrimp lethality assay. Bangladesh Journal of Pharmacology: 186-189

46. Ramazani A., Sardari S., Zakeri S., Vaziri B. (2010). In vitro antiplasmodial and phytochemical study of five Artemisia species from Iran and in vivo activity of two species. Parasitology Research: 593-599.

47. Ravikumar S., Jacob Inbaneson S., Suganthi P., Gnanadesigan M. (2011a). In vitro antiplasmodial activity of ethanolic extracts of mangrove plants from South East coast of India against chloroquinesensitive Plasmodium falciparum. Parasitology Research: 873-878.

48. Ravikumar S., Ramanathan G., Jacob Inbaneson S., Ramu A. (2011b). Antiplasmodial activity of two marine polyherbal preparations from Chaetomorpha antennina and Aegiceras corniculatum against Plasmodium falciparum. Parasitology Research: 107-113.

49. Ravikumar S., Inbaneson J.S., Suganthi P., Gokulakrishnan R., Venkatesan M. (2011c). In vitro antiplasmodial activity of ethanolic extracts of Seaweed macroalgae against Plasmodium falciparum. Parasitology Research: 1411-1416.

50. Saliba L.J., Krzyz R.M. (1966). Effect of heavy metals on hatching of brine-shrimp eggs. Marine Pollution Bulletin: 181-82.

51. Sam T.W. (1993). Toxicity testing using the brine shrimp: Artemia salina. In: Colegate, S. M. and Molyneux, R. J. (Eds.), Bioactive Natural Products Detection, Isolation, and Structural Determination. CRC Press, Boca Raton: 442-456.

52. Sanon S., Azas N., Gasquet M., Ollivier E., Mahiou V., Barro N., Cuzin-Ouattara N., Traore A.S., Esposit F., Balansard G., TimonDavid P. (2003a). Antiplasmodial activity of alkaloid extracts from Pavetta crassipes (K. Schum) and Acanthospermum hispidum (DC), two plants used in traditional medicine in Burkina Faso. Parasitology Research: 314-317.

53. Sanon S., Ollivier E., Azas N., Mahiou V., Gasquet M., Ouattara C.T., Nebie I., Traore A.S., Esposito F., Balansard G., Timon-David P., Fumoux F. (2003b). Ethnobotanical survey and in vitro antiplasmodial activity of plants used in traditional medicine in Burkina Faso. Journal of Ethnopharmacology: 143-147.

54. Somayeh E., Azadeh G., Farzaneh N., Mahmoud M. (2015). Antiplasmodial Activity and Cytotoxicity of Plants Used in Traditional 
Medicine of Iran for the Treatment of Fever. Iranian Journal of Pharmaceutical Research: 103-107

55. Sufian M., Haque M. (2015). Cytotoxic, thrombolytic, membrane stabilizing and anti-oxidant activities of Hygrophila schulli. Bangladesh Journal of Pharmacology: 692-96.

56. Syahmi A.R.M., Vijayaratna S., Sasidharan S., Latha L.Y., Kwan Y.P., Lau Y.L., Shin L.N., Chen Y. (2010). Acute oral toxicity and brine shrimp lethality of Elaeis guinennsis Jacq. (oil palm leaf) methanol extract. Molecules: 8111-21.

57. Tona L., Cimanga R.K., Mesia K., Musuamba C.T., De Bruyne T., Apers S., Hernans N., Van M.S., Pieters L., Totté J., Vlietinck A.J. (2004). In vitro antiplasmodial activity of extracts and fractions from seven medicinal plants used in the Democratic Republic of Congo. Journal of Etnopharmacology: 27-32.

58. Tona L., Ngimbi N.P., Tsakala M., Mesia K., Cimanga K., Apers S., De Bruyne T., Pieters L., Totte J., Vlietinck A.J. (1999). Antimalarial activity of 20 crude extracts from nine African medicinal plants used in Kinshasa, Congo. Journal of Ethnopharmacology: 193- 203.

59. Trager W., Jensen J. (1976). Human malaria parasites in continuous culture. Science: 673-675.

60. Weniger B., Lagnika L., Vonthron-Sénécheau C., Adjobimey T., Gbenou J., Moudachirou M., Brun R., Anton R., Sanni A. (2004). Evaluation of ethnobotanically selected Benin medicinal plants for their in vitro antiplasmodial activity. Journal of Ethnopharmacology: 279-284.

61. Wongsrichanalai C., Webster H.K., Wimonwattrawatee T., Sookto P., Chuanak N., Thimasarn K., Wernsdorfer W.H. (1992a). Emergence of multi-resistant Plasmodium falciparum in Thailand: in vitro tracking. The American Journal of Tropical Medicine Hygiene: 112-116.

62. Wongsrichanalai C., Webter H.K., Wimonmattrawatee T., Sookto P., Chuanak N., Tiamasam K., Werndorfer W.H. (1992b). In vitro sensitivy of Plasmodium falciparum isolates in Thailand to quinine and chloroquine, 1984-1990. Southeast Asian Journal of Tropical Medicine Public Health: 533-536.

63. Wright C.W., Bray D.H., O’Neill M.J., Warhurst D.C., Phillipson J.D., Quetin-Leclercq J., Angenot L. (1991). Antiamoebic and antiplasmodial activities of alkaloids isolated from Strychnos usambarensis. Planta Medica: 337-340.

64. Wright C.W., Addae-Kyereme J., Breen A.G., Brown J.E., Cox M.F., Croft S.L., Gokcek Y., Kendrick H., Phillips R.M., Pollet P.L. (2001). Synthesis and evaluation of cryptolepine analogues for their potential 
as new antimalarial agents. Journal of Medicinal Chemistry: 31873194.

65. Wright C.W. (2002). Antiprotozoal natural products. In Pharmacognosy, 15th Ed. Evans, W.C., Ed. Harcourt, London, pp. 407-413.

66. Zirihi G.N., Mambu L., Guede-Guina F., Bodo B., Grellier P. (2005). In vitro antiplasmodial activity and cytotoxicity of 33 West African plants used for treatment of malaria. Journal of Ethnopharmacology: 281-285. 